\title{
Left Ovary
}

National Cancer Institute

\section{Source}

National Cancer Institute. Left Ovary. NCI Thesaurus. Code C32969.

The ovary that is located in the left side of the pelvis. 